\title{
A prospective clinical and radiographic 12-month outcome study of patients undergoing single-level anterior cervical discectomy and fusion for symptomatic cervical degenerative disc disease utilizing a novel viable allogeneic, cancellous, bone matrix (trinity evolution $^{\mathrm{TM}}$ ) with a comparison to historical controls
}

\author{
Jed Vanichkachorn $^{1} \cdot$ Timothy Peppers $^{2} \cdot$ Dennis Bullard $^{3} \cdot$ Scott K. Stanley $^{4}$. \\ Raymond J. Linovitz ${ }^{5}$ James T. Ryaby ${ }^{6}$
}

Received: 13 April 2015/Revised: 11 January 2016/Accepted: 15 January 2016/Published online: 5 February 2016 (C) The Author(s) 2016. This article is published with open access at Springerlink.com

\begin{abstract}
Purpose This multicenter clinical study was performed to assess the safety and effectiveness of Trinity Evolution ${ }^{\circledR}$ (TE), a viable cellular bone allograft, in combination with a PEEK interbody spacer and supplemental anterior fixation in patients undergoing anterior cervical discectomy and fusion (ACDF).

Methods In a prospective, multi-center study, 31 patients that presented with symptomatic cervical degeneration at one vertebral level underwent ACDF with a PEEK interbody spacer (Orthofix, Inc., Lewisville, TX, USA) and supplemental anterior fixation. In addition all patients had the bone graft substitute, Trinity Evolution (Musculoskeletal Transplant Foundation, Edison, NJ, USA), placed within the interbody spacer. At 6 and 12 months, radiographic fusion was evaluated as determined by independent radiographic review of angular motion $\left(\leq 4^{\circ}\right)$ from flexion/extension $\mathrm{X}$-rays combined with presence of bridging bone across the adjacent endplates on thin cut $\mathrm{CT}$
\end{abstract}

Raymond J. Linovitz

RayLinovitz@gmail.com

1 Tuckahoe Orthopaedic Associates, 1501 Maple Ave., Richmond, VA 23226, USA

2 Seaside Spine Medical Associates, 320 Santa Fe Dr., Suite 300, Encinitas, CA 92024, USA

3 Triangle Neurosurgery, 1540 Sunday Dr., Raleigh, NC 27607, USA

4 Denver-Vail Orthopedics, P.C., 8101 E. Lowry Blvd., Suite 260, Denver, CO 80230, USA

5 PO Box 1671, Rancho Santa Fe, CA 92067, USA

6 Orthofix, Inc., 3451 Plano Parkway, Lewisville, TX 75056, USA scans. In addition other metrics were measured including function as assessed by the Neck Disability Index (NDI), and neck and arm pain as assessed by individual Visual Analog Scales (VAS).

Results The fusion rate for patients using a PEEK interbody spacer in combination with TE was $78.6 \%$ at 6 months and $93.5 \%$ at 12 months. When considering high risk factors, 6-month fusion rates for patients that were current or former smokers, diabetic, overweight or obese/extremely obese were $70 \%(7 / 10), 100 \%(1 / 1)$, $70 \%(7 / 10)$, and $82 \%(9 / 11)$, respectively. At 12 months, the fusion rates were $100 \%$ (12/12), $100 \%(2 / 2), 100 \%$ (11/11) and $85 \%$ (11/13), respectively. Neck function, and neck/arm pain were found to significantly improve at both time points. No serious allograft related adverse events occurred and none of the 31 patients had subsequent additional cervical surgeries.

Conclusions Patients undergoing single-level ACDF with TE in combination with a PEEK interbody spacer and supplemental anterior fixation had a high rate of fusion success without serious allograft-related adverse events.

Keywords ACDF - PEEK cage $\cdot$ Allograft $\cdot$ Cervical spine $\cdot$ Spine fusion

\section{Introduction}

Since the development of the anterior approach for anterior cervical discectomy and fusion (ACDF) [1] many different combinations of graft materials and interbody spacer devices have been explored. This includes autografts [2], allografts [3], and interbody spacers made from polyetherether-ketone (PEEK) [4], and porous tantalum [5]. Autografts have traditionally been considered the gold standard 
due to high fusion rates, although there are disadvantages associated with autograft use such as donor site morbidity, additional time in the operating room, and uncertain quality of the patient's own bone [6, 7]. Despite these drawbacks, autograft has remained the preferred choice since its usage has been reported to be associated with a higher fusion rate than allograft. For example, a recent meta-analysis for oneand two-level stand-alone ACDF comparing autograft and allograft showed a higher fusion rate for autograft for both single level and multi-level ACDF cases at 12 months (pooled results 92 vs. $78 \%$ ) [3]. Although no clinical superiority was established, allograft ACDFs were found to have delayed time to fusion [3]. Conversely, other studies have found no difference in fusion rates between autografts and allografts for multi-level ACDFs using rigid plate fixation [8].

PEEK ACDF devices are gaining acceptance as the new standard for treatment of cervical disc disease [9], as they show similar fusion rates as autografts $[10,11]$. PEEK cages for ACDF surgeries have the added advantages of increased cervical lordosis, immediate biomechanical support, increased intervertebral height and easy fusion assessment through X-ray and/or CT. In order to aid in fusion for high risk patients PEEK interbody devices are often combined with autografts, allografts or bone graft substitutes such as hydroxyapatite for single and multi-level ACDFs [12-16]. While there currently are a number of products on the market to minimize or replace the use of autografts for ACDFs, few of these products contain all three essential bone-forming elements of autografts (osteogenicity, osteoconductivity, and osteoinductivity [17]) in a single, standalone product. Trinity Evolution ${ }^{\circledR}$ (TE) is a cryopreserved allograft that consists of viable cellular cancellous bone matrix and demineralized cortical bone. It possesses all three of the key properties for successful bone grafting (osteogenic cells including mesenchymal stem cells and osteoprogenitors, osteoinductive proteins, and an osteoconductive matrix [18]) and can be considered to be a practical alternative to autograft. TE has the advantages of having more predictable quality than autograft bone in cases where the source of the autograft would be from high risk donors. TE is only produced from healthy donors that are strictly screened and carefully processed to maintain the viability of endogenous osteogenic cells and the osteoinductivity of the demineralized cortical component. The purpose of this multicenter clinical study was to assess the safety and effectiveness of the TE viable cellular bone allograft in combination with a PEEK interbody spacer in cervical arthrodesis.

\section{Materials and methods}

\section{Study population}

From October 2009 to June 2012, a prospective, multicenter, study was conducted at 4 investigational sites to evaluate Trinity Evolution ${ }^{\circledR}$ in combination with a PEEK interbody spacer for ACDF surgery. All patients with symptomatic cervical degeneration at one vertebral level between $\mathrm{C} 3$ and $\mathrm{T} 1$ were eligible for the study and those enrolled underwent ACDF with an Orthofix PEEK interbody spacer (Orthofix, Inc., Lewisville, TX, USA) and supplemental anterior fixation. Patients who had previously undergone a cervical fusion were included (except if the prior interbody surgery was at the same level). IRB approval was obtained for each site prior to the initiation of enrollment. Exclusion criteria encompassed the use of any other bone graft or bone graft substitute in addition to or in place of Trinity Evolution in and around the interbody spacer. Patients were examined pre-operatively, at 6 months ( \pm 1 month), and at 12 months ( \pm 2 months). A total of 31 patients were found eligible for the study (Table 1). The 31 patients received single level fusions at the vertebral locations between $\mathrm{C} 3 / \mathrm{C} 4$ and $\mathrm{C} 6 / \mathrm{C} 7$ with Trinity Evolution being placed within and around each cage (Table 2). All 31 patients were evaluated for primary and secondary endpoints at both 6 and 12 months.

\section{Primary endpoint}

The primary endpoint was radiographic fusion status based on independent review of $\mathrm{CT}$ scans and radiographic review of angular motion (Medical Metrics Inc., Houston, TX, USA). Specifically the criteria for fusion was the presence of bridging bone across the adjacent endplates on thin cut CT scans with sagittal and coronal reconstructions in addition to $\leq 4^{\circ}$ angular motion from flexion/extension $\mathrm{X}$-rays (Fig. 1).

\section{Secondary endpoints}

Secondary endpoints included function as assessed by the Neck Disability Index (NDI), and neck and arm pain as assessed by individual Visual Analog Scales (VAS). Based on the literature, the following changes to the examined parameters were considered clinically significant improvements [19]:

1. A change in NDI of more than $17.3 \%$ points

2. A change in VAS arm pain of more than $41 \mathrm{~mm}$

3. A change in VAS neck pain of more than $26 \mathrm{~mm}$ 
Table 1 Patient demographics

\begin{tabular}{ll}
\hline Patients & $n$ \\
\hline Male & 12 \\
Female & 19 \\
Total & Years \\
\hline Age & $48.9 \pm 8.1$ \\
\hline Mean \pm SD & $48 / 30 / 68$ \\
Median/Minimum/Maximum & $n(\%)$ \\
\hline Age group & $16(51.6)$ \\
\hline$n<50$ years & $30(96.8)$ \\
$n<65$ years & $n(\%)$ \\
\hline Smoking status & $19(61.2)$ \\
\hline Never used tobacco & $5(16.1)$ \\
Currently using tobacco & $n(\%)$ \\
\hline Diabetic & $29(93.5)$ \\
\hline No & $2(6.5)$ \\
Yes & $n(\%)$ \\
\hline Weight status (based on BMI) & $7(22.6)$ \\
\hline Normal weight & $11(35.5)$ \\
Overweight & $11(35.5)$ \\
Obese & $2(6.5)$ \\
\hline Prior adjacent level fusion? & $3(9.7)$ \\
\hline No & \\
\hline
\end{tabular}

Table 2 Surgical information

\begin{tabular}{lc}
\hline Vertebral location of fusion & $n(\%)$ \\
\hline C3-C4 & $1(3.2)$ \\
C4-C5 & $4(12.9)$ \\
C5-C6 & $16(51.6)$ \\
C6-C7 & $10(32.3)$ \\
\hline
\end{tabular}

\section{Adverse events}

All adverse events were collected per protocol during the evaluation period. Following the study all adverse events were adjudicated by an independent medical consultant, which included tissue processor cross referencing of the allograft lot numbers associated with the adverse event to determine if the lot had previously been associated with any other adverse event.

\section{Statistical analysis}

Fusion is presented as the percentage of patients fused. Secondary parameters are presented as the mean and standard error (SE). A multiple paired $t$ test with a subsequent Bonferroni correction was done to determine if changes from baseline in the secondary outcomes measures were significant. The statistical analyses were performed using SAS (version 9.3, Cary, NC, USA). Significance was set at $p<0.05$.

\section{Results}

Of the 31 patients, 28 completed their 6-month study visit, while all 31 completed their 12 -month study visit.

\section{Fusion}

For this study, the fusion rate for patients using Orthofix PEEK interbody spacers in combination with the allograft trinity evolution, was $78.6 \%$ at 6 months and $93.5 \%$ at 12 months. Specifically, when considering high risk factors 6-month fusion rates for patients that were current or former smokers, diabetic, overweight or obese/extremely obese were $70 \%(7 / 10), 100 \%(1 / 1), 70 \%(7 / 10)$, and $82 \%$ (9/11), respectively. At 12 months, the fusion rates were $100 \%$ (12/12), $100 \%$ (2/2), $100 \%$ (11/11) and $85 \%$ (11/13), respectively.

\section{Secondary endpoints}

Mirroring the primary endpoint, neck function was found to significantly improve from baseline to 6 months $(p<0.0001)$. In addition, neck function was significantly improved from 6 to 12 months $(p<0.0040)$ (Fig. 2). Similarly, both neck and arm (right and left) pain decreased significantly at 6 and 12 months relative to pre-op assessments (Fig. 3).

\section{Adverse events}

During the time course of the study the 31 patients encountered a total of 26 distinct adverse events (AEs), which were specific to 16 distinct patients. These adverse events included carpal tunnel syndrome, minor pain, numbness, permanent and/or unresolved pain, and swelling. Subsequent medical adjudication of the 26 adverse events found that no AEs were definitely or probably related to Trinity Evolution. However, five AEs were found to be possibly related to Trinity Evolution with three of them of mild severity and two of moderate severity. Specifically two were related to permanent and/or unresolved pain, two were related to numbness and one was 

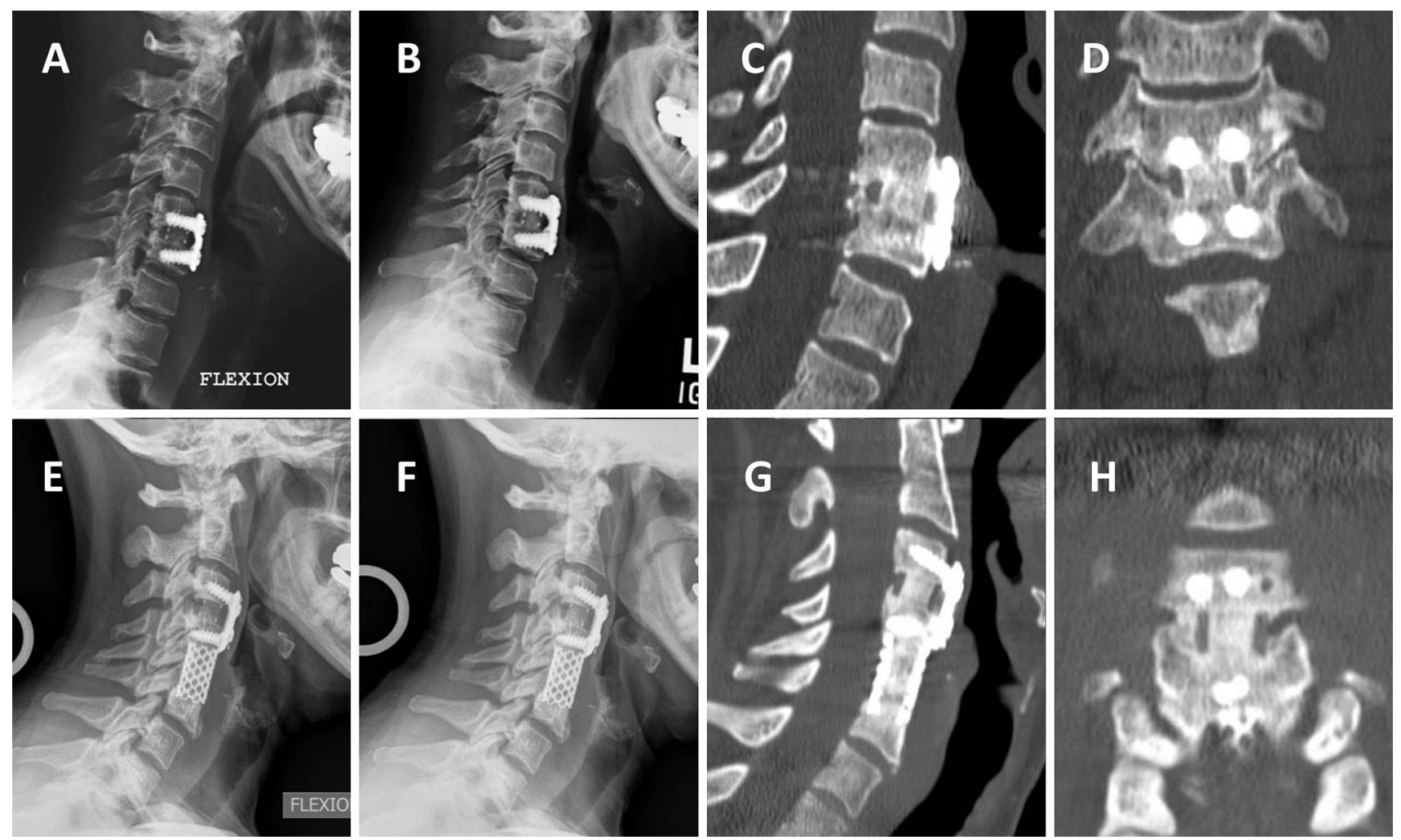

Fig. 1 Representative lateral cervical spine radiographs (flexion) and coronal and sagittal CT scans for two patients with single level ACDF using a PEEK cage with Trinity Evolution allograft showing solid

fusion at C5-C6 (a, b radiographs at 6 and 12 months, respectively. c, d CT scan at 12 months), and at $\mathrm{C} 3-\mathrm{C} 4$ (e, f radiographs at 6 and 12 months, respectively. $\mathbf{g}$, h CT scan at 12 months)

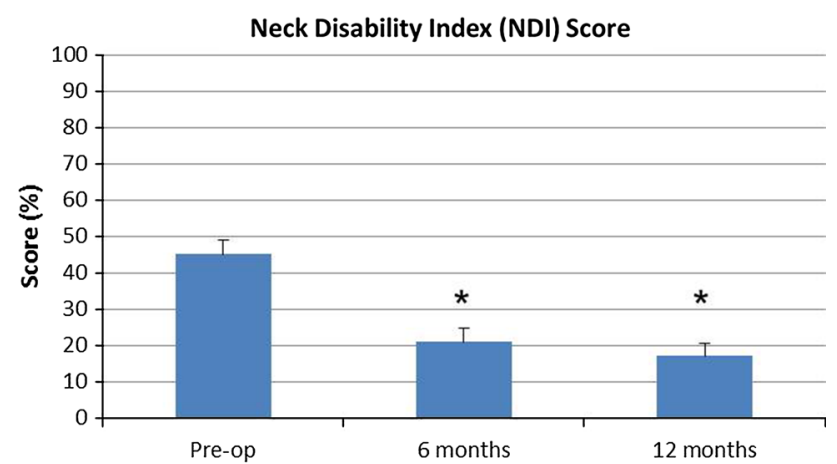

Fig. 2 Neck disability index (NDI) scores. Asterisk significant difference relative to pre-op $(p<0.05)$

related to posterior neck pain. Two of these AE's resolved within 30 days of recording, while the remaining persisted intermittently following the study. None of the 31 patients had subsequent additional cervical surgeries.

\section{Discussion}

When conservative care fails to alleviate the pain and neurological deficits caused by degenerative disc disease in the cervical spine, the most common recourse is surgical

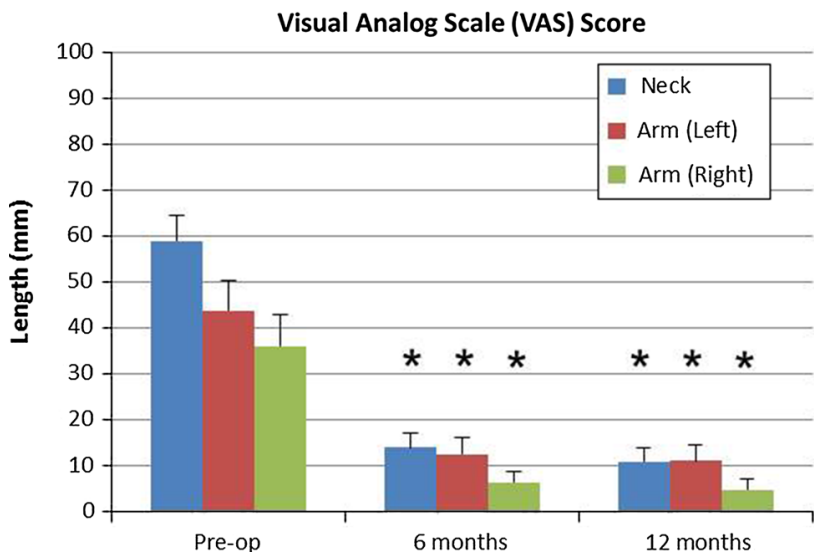

Fig. 3 Assessment of neck and arm pain using VAS. Asterisk significant difference relative to pre-op $(p<0.05)$

decompression of the affected nerves and/or spinal cord. Decompression is often accomplished via an anterior approach whereby essentially the entire disc as well as any bony osteophytes and ligaments that are compressing the spinal cord and/or nerves are removed. While usually successful at decompressing affected neural structures, the decompression often results in collapse of the disc space, instability and recurrent symptomatology. As mentioned previously, most anterior cervical decompressions are 
therefore followed by insertion of a structural interbody spacer such as an autograft, allograft, or a synthetic spacer filled with a bone graft. As the results indicate, TE in combination with a PEEK interbody spacer is a viable option which led to a $94 \%$ fusion rate in this study at the final time point based on radiographic evidence of motion and bony bridging. This fusion rate, although not statistically evaluated, is comparable to that reported for single level ACDFs using standalone autografts $(97 \%)$ and other allografts $(87 \%)$ at 12 months per radiographic evidence [3]. In addition the results compare favorably to fusion rates for other single level ACDF studies using PEEK cages filled with hydroxyapatite (61 and $100 \%$ at 6 and 12 months, respectively) [12], various allografts (100\% at 10-12 months, but not including higher risk patients) [13, 14], and carbon fiber reinforced polymer (CFRP) packed with bone marrow-saturated collagen/hydroxyapatite wafers (100\% at 12 months) [20]. In addition, some of these studies $[3,14]$ only used bridging bone as the fusion criteria thus potentially achieving more favorable fusion rates compared to a more stringent criteria.

As evident by the many studies mentioned there remains an intense search for an autograft replacement which will encompass the three important autograft components, namely viable osteogenic cells, an osteoconductive matrix and osteoinductive growth factors. In addition to the referenced potential clinical solutions to this problem some pre-clinical studies [21] have also examined the use of degradable radiolucent cage filler (PLCL) in PEEK cages with or without adipose stem cells or autograft. This study showed no enhancement of the rate and number of interbody fusions, but with a trend towards superior results with autograft, indicating that all three autograft components may indeed be needed to achieve an autograft alternative.

When stratifying the 31 patients into high-risk groups it was found that the fusion rates ranging from 70 to $100 \%$ at 6 months to $85-100 \%$ at 12 months were still comparable to the literature where no high-risk patients were included, in particular at 12 months (92-100 \%) [10, 11, 13, 14, 22]. Having a comparable fusion rates to other PEEK/allograft ACDF studies that have less stringent fusion criteria and do not include a majority of high risk patients indicates that Trinity Evolution may help negate any physiological barrier to fusion during ACDF that is associated with high risk factors. In addition, the current study also compared favorable to other high-risk patient studies where smokers underwent ACDF with allograft and anterior plating (100 vs. $91 \%$ at 12 months) [22].

The NDI results indicated a significant mean reduction over time with a 53 and $62 \%$ drop from pre-op to 6 and 12 months, respectively. However, based on the set criteria in the literature the changes seen on an individual basis were only clinically significant for 68 and $74 \%$ of the patients at 6 and 12 months, respectively [19]. Despite this, these results do parallel those found by Faldini et al. at 6 months (61\% mean reduction in NDI) [14]. Similarly the mean VAS neck scores showed significant changes over time, but individual scores indicated that only 64 and $74 \%$ of the patients saw clinically significant changes at 6 and 12 months, respectively. Although a clear correlation between fusion status and NDI/VAS scores is not found, the results are similar to those found in the literature despite these studies being done without including a majority of high risk patients [14]. This favorable comparison and the fact that $75 \%$ of the patients in this study had at least one high risk factor indicates that TE may help promote fusion during ACDF even for high risk patients.

Finally, while 26 adverse events were encountered during the course of this study, only five of these were deemed possibly related to Trinity Evolution, and were only categorized as of mild (three) or moderate (two) severity, with two of these resolving within 30 days. This in combination with the fact that no subsequent cervical surgeries were performed on any of the 31 subjects indicates that Trinity Evolution is safe to use as an interbody filler during single-level cervical fusion. The study has the limitation of a low number of patients both overall and for some of the stratified high risk groups. However, a few of the high risk groups consisted of at least ten patients enabling the reader to still evaluate the potential for the novel allograft. Despite this, Trinity Evolution can also be evaluated irrespectively of any of the stratified groups, which, as it has been shown, still reveals positive results.

In conclusion, patients who received Trinity Evolution in combination with a PEEK interbody device during single level cervical fusion surgery had a high rate of fusion success both overall and when stratified into high risk groups, while having no serious allograft related adverse events.

Acknowledgments The authors thank Sannah Ladiwalla, MS, Rebekah Hahn, PhD and Erik Waldorff, PhD, for their help reviewing, statistical analysis and manuscript organization.

\section{Compliance with ethical standards}

Conflict of interest One of the authors (JTR) is employed by and own stock in Orthofix, Inc., Lewisville, TX. In addition, the authors JV, TP, DB, SKS and RJL are independent consultants for Orthofix, Inc., Lewisville, TX, USA.

Open Access This article is distributed under the terms of the Creative Commons Attribution 4.0 International License (http://crea tivecommons.org/licenses/by/4.0/), which permits unrestricted use, distribution, and reproduction in any medium, provided you give appropriate credit to the original author(s) and the source, provide a link to the Creative Commons license, and indicate if changes were made. 


\section{References}

1. Smith GW, Robinson RA (1958) The treatment of certain cervical-spine disorders by anterior removal of the intervertebral disc and interbody fusion. J Bone Joint Surg Am 40-A:607-624

2. Anderson DG, Albert TJ (2002) Bone grafting, implants, and plating options for anterior cervical fusions. Orthop Clin North Am 33:317-328

3. Floyd T, Ohnmeiss D (2000) A meta-analysis of autograft versus allograft in anterior cervical fusion. Euro Spine J Off Publ Euro Spine Soc Euro Spinal Deform Soc Euro Sect Cerv Spine Res Soc 9:398-403

4. Lemcke J, Al-Zain F, Meier U, Suess O (2011) Polyetheretherketone (PEEK) spacers for anterior cervical fusion: a retrospective comparative effectiveness clinical trial. Open Orthop J 5:348-353. doi:10.2174/1874325001105010348

5. Lofgren H, Engquist M, Hoffmann P, Sigstedt B, Vavruch L (2010) Clinical and radiological evaluation of Trabecular Metal and the Smith-Robinson technique in anterior cervical fusion for degenerative disease: a prospective, randomized, controlled study with 2-year follow-up. Euro Spine J Off Publ Euro Spine Soc Euro Spinal Deform Soc Euro Sect Cerv Spine Res Soc 19:464-473. doi:10.1007/s00586-009-1161-z

6. Samartzis D, Shen FH, Goldberg EJ, An HS (2005) Is autograft the gold standard in achieving radiographic fusion in one-level anterior cervical discectomy and fusion with rigid anterior plate fixation? Spine 30:1756-1761

7. Silber JS, Anderson DG, Daffner SD, Brislin BT, Leland JM, Hilibrand AS, Vaccaro AR, Albert TJ (2003) Donor site morbidity after anterior iliac crest bone harvest for single-level anterior cervical discectomy and fusion. Spine 28:134-139. doi:10.1097/01.BRS.0000041587.55176.67

8. Samartzis D, Shen FH, Matthews DK, Yoon ST, Goldberg EJ, An HS (2003) Comparison of allograft to autograft in multilevel anterior cervical discectomy and fusion with rigid plate fixation. Spine J Off J North Am Spine Soc 3:451-459

9. Cho DY, Liau WR, Lee WY, Liu JT, Chiu CL, Sheu PC (2002) Preliminary experience using a polyetheretherketone (PEEK) cage in the treatment of cervical disc disease. Neurosurgery 51:1343-1349 (discussion 1349-1350)

10. Zhou J, Xia Q, Dong J, Li X, Zhou X, Fang T, Lin H (2011) Comparison of stand-alone polyetheretherketone cages and iliac crest autografts for the treatment of cervical degenerative disc diseases. Acta Neurochir (Wien) 153:115-122. doi:10.1007/ s00701-010-0821-4

11. Chou YC, Chen DC, Hsieh WA, Chen WF, Yen PS, Harnod T, Chiou TL, Chang YL, Su CF, Lin SZ, Chen SY (2008) Efficacy of anterior cervical fusion: comparison of titanium cages, polyetheretherketone (PEEK) cages and autogenous bone grafts. J Clin Neurosci Off J Neurosurg Soc Austral 15:1240-1245. doi:10.1016/j.jocn.2007.05.016
12. Mastronardi L, Ducati A, Ferrante L (2006) Anterior cervical fusion with polyetheretherketone (PEEK) cages in the treatment of degenerative disc disease. Preliminary observations in 36 consecutive cases with a minimum 12-month follow-up. Acta Neurochirurg 148:307-312. doi:10.1007/s00701-005-0657-5 (discussion 312)

13. Liao JC, Niu CC, Chen WJ, Chen LH (2008) Polyetheretherketone (PEEK) cage filled with cancellous allograft in anterior cervical discectomy and fusion. Int Orthop 32:643-648. doi:10. 1007/s00264-007-0378-x

14. Faldini C, Chehrassan M, Miscione MT, Acri MT, d'Amato M, Pungetti c, Luciani D, Giannini S (2011) Single-level anterior cervical discectomy and interbody fusion using PEEK anatomical cervical cage and allograft bone. J Orthop Traumatol 12:201-205

15. Topuz K, Colak A, Kaya S, Simsek H, Kutlay M, Demircan MN, Velioglu M (2009) Two-level contiguous cervical disc disease treated with peek cages packed with demineralized bone matrix: results of 3-year follow-up. Euro Spine J Off Publ Euro Spine Soc Euro Spinal Deform Soc Euro Sect Cerv Spine Res Soc 18:238-243. doi:10.1007/s00586-008-0869-5

16. Kersten RF, van Gaalen SM, de Gast A, Oner FC (2013) Polyetheretherketone (PEEK) cages in cervical applications: a systematic review. Spine J Off J North Am Spine Soc. doi:10. 1016/j.spinee.2013.08.030

17. Vaccaro AR (2002) The role of the osteoconductive scaffold in synthetic bone graft. Orthopedics 25:s571-s578

18. Rush SM (2010) Trinity Evolution: mesenchymal stem cell allografting in foot and ankle surgery. Foot Ankle Special 3:140-143. doi:10.1177/1938640010369638

19. Parker SL, Godil SS, Shau DN, Mendenhall SK, McGirt MJ (2013) Assessment of the minimum clinically important difference in pain, disability, and quality of life after anterior cervical discectomy and fusion: clinical article. J Neurosurg Spine 18:154-160. doi:10.3171/2012.10.SPINE12312

20. Chen F, He W, Mahaney K, Noeller J, Mhanna N, Viljoen S, Torner J, Hitchon P (2013) Alternative grafts in anterior cervical fusion. Clin Neurol Neurosurg 115:2049-2055. doi:10.1016/j. clineuro.2013.07.013

21. Kroeze RJ, Smit TH, Vergroesen PP, Bank RA, Stoop R, van Rietbergen B, van Royen BJ, Helder MN (2015) Spinal fusion using adipose stem cells seeded on a radiolucent cage filler: a feasibility study of a single surgical procedure in goats. Euro Spine J Off Publ Euro Spine Soc Euro Spinal Deform Soc Euro Sect Cerv Spine Res Soc 24:1031-1042. doi:10.1007/s00586014-3696-x

22. Luszczyk M, Smith JS, Fischgrund JS, Ludwig SC, Sasso RC, Shaffrey CI, Vaccaro AR (2013) Does smoking have an impact on fusion rate in single-level anterior cervical discectomy and fusion with allograft and rigid plate fixation? Clinical article. J Neurosurg Spine 19:527-531. doi:10.3171/2013.7.SPINE13208 